\title{
Autistic-Like Traits, Sociosexuality, and Hormonal Responses to Socially Stressful and Sexually Arousing Stimuli in Male College Students
}

\author{
Davide Ponzi ${ }^{1,2}$ - Andrea Henry ${ }^{2}$. \\ Konrad Kubicki ${ }^{2}$ - Nora Nickels ${ }^{2}$. \\ M. Claire Wilson ${ }^{2} \cdot$ Dario Maestripieri $^{2}$
}

Received: 4 June 2015 /Revised: 22 August 2015 / Accepted: 26 August 2015 /

Published online: 22 September 2015

(C) Springer International Publishing 2015

\begin{abstract}
We tested the hypothesis that autistic-like traits are associated with restricted sociosexuality and investigated the role of stress and sex hormones as potential physiological mechanisms underlying this association. Study participants were heterosexual male college students $(n=107)$. Individuals with more autistic-like traits reported lower short-term mating orientation and less previous sexual experience. Autistic-like traits were associated with greater cortisol secretion during a Trier Social Stress Test (TSST) and greater testosterone and cortisol reactivity to a video with explicit erotic content. Cortisol secretion during the TSST was negatively correlated with short-term mating orientation. A mediation analysis suggested that cortisol reactivity to socially stressful stimuli may be one of the physiological mechanisms linking autistic-like traits and sociosexuality. These findings have implications for our understanding of autisticlike traits in normal populations, our knowledge of the relationship between psychosocial stress, sexual arousal, and sociosexuality, and also for research on the role of hormonal mechanisms in inter-individual variation in male life history strategies.
\end{abstract}

Keywords Personality $\cdot$ Sociosexuality $\cdot$ Life history $\cdot$ Hormones $\cdot$ Sex $\cdot$ Stress

\section{Introduction}

Autistic-like traits are expressions of normal personality variation in individuals with normal intelligence (Baron-Cohen et al. 2001; Wheelwright et al. 2010; White et al.

Davide Ponzi

ponzdbiol@gmail.com

1 Department of Anthropology, University of Utah, 270 S 1400 E, Salt Lake City, UT 84112, USA

2 Institute for Mind and Biology, The University of Chicago, Chicago, IL 60637, USA 
2011). They include in milder form many behavioral and cognitive aspects of Autism Spectrum Disorders, can be quantified with a self-administered questionnaire, the Autism-Spectrum Quotient (AQ; Baron-Cohen et al. 2001), and have been shown to be substantially heritable (Hoekstra et al. 2007). The AQ assesses different psychological and behavioral manifestations of autistic-like traits such as poor communicative or social skills, reduced attention switching and repetitive behavior, greater interest in patterns and details, and low imagination. These different facets of autistic-like traits are correlated but also exhibit independent variation, and probably have distinct genetic bases and are regulated by different neurobiological mechanisms (see Del Giudice et al. 2010; Happé and Ronald 2008).

Several hypothetical scenarios have been proposed for the evolution or maintenance of these traits in human populations (e.g., Del Giudice et al. 2010; Reser 2011; Shaner et al. 2008). In one of these models, Del Giudice et al. (2010) argued that, regardless of the initial reasons for the evolution of autistic-like traits, their maintenance in human populations can be explained by their effects on mating- and parenting-related behaviors and that such effects are best understood from a life-history perspective. Life history theory is a branch of evolutionary biology that addresses the way organisms allocate time and energy to the various activities that comprise their life cycle (e.g., Stearns 1992). In humans, much inter-individual variation in growth and sexual maturation as well as in sociosexual behavior can be functionally explained in terms of distinct survival and reproductive strategies such as those collectively known as slow and fast life histories. Slow life histories are characterized by trade-offs favoring maintenance over growth, future over current reproduction (e.g., delayed sexual maturation), monogamous rather than promiscuous sexual relationships, and offspring quality over quantity (i.e., low offspring number and high parental investment). In contrast, individuals who adopt fast life-history strategies are more likely to sexually mature and to start mating early in life, mate frequently, and invest relatively little in relationships and children (e.g., Kaplan and Gangestad 2005).

Del Giudice et al. (2010) hypothesized that autistic-like traits contribute to a slow life history phenotype geared toward low mating effort, high parental investment, and long-term allocation of resources. In their view, autistic-like traits are maintained in human populations through sexual selection processes involving mate choice (especially female choice for males with autistic-like traits). Autistic-like traits can be expected to promote relational stability through a preference for routines and a predictable lifestyle, reduced novelty-seeking and risk-taking, and low interest in potential alternative partners. Consistent with this hypothesis, people high in autistic-like traits have reported longer duration of romantic relationships (Jobe and White 2007), stronger commitment to long-term relationships and increased investment of time and resources in one's romantic partner, coupled with restricted sociosexuality and fewer sexual partners (Del Giudice et al. 2010).

Life history theory assumes that individual differences in sociosexual behavior are part of larger clusters of integrated behavioral, psychological, and physiological traits including competitiveness and aggressiveness, cooperation and altruism, impulsivity, sensation-seeking, risk-taking, attachment styles, and physiological reactivity to stressful or sexual stimuli (e.g., Del Giudice 2014; Kaplan and Gangestad 2005). Thus, the different sociosexual propensities characteristic of slow and fast life history phenotypes are likely to be mediated by particular psychological and/or physiological mechanisms 
(e.g., low vs high sensation-seeking and risk-taking, low vs high physiological arousability in response to stress or sexual stimuli). Consistent with this, Del Giudice et al. (2014) recently found that high autistic-like traits and restricted sociosexuality were also associated with low impulsivity and sensation-seeking. These associations, however, were not explained by correlations between autistic-like traits and broad personality traits such as extraversion and openness to experience, corroborating the idea that autistic-like traits are functionally distinct from other dimensions of personality.

Two likely candidates as potential physiological mechanisms underlying the association between autistic-like traits and restricted sociosexuality are sex hormones (e.g., testosterone and estrogen) and stress hormones (e.g., cortisol). The possible role of sex hormones is suggested by multiple lines of evidence. First, autistic-like traits are more prevalent in males than in females, and sex hormones are implicated in many anatomical, physiological, and behavioral differences between males and females (BaronCohen 2003). Second, within-sex variation in sociosexuality is generally accompanied by variation in sex hormone profiles, such that, for example, single and sexually promiscuous men have higher circulating testosterone than paired and sexually monogamous men (e.g., Maestripieri et al. 2014). Finally, sex hormones play an important role in sexual motivation, sexual desire, and sexual arousability (e.g., Goldey and van Anders 2015; Roney and Simmons 2013) and variation in the baseline concentrations of these hormones or in their reactivity to sexual stimuli may account for variation (both across individuals and within individuals over time) in interest in casual sex or willingness to engage in courtship behavior (e.g., Maestripieri et al. 2014; Roney et al. 2003; Roney and Simmons 2013).

Physiological arousability to socially stressful stimuli can play an important role in mediating the association between autistic-like traits and restricted sociosexuality because transient or chronic elevation in stress hormones can suppress sex hormones and sexual motivation (e.g., Doerr and Pirke 1976). Therefore, if individuals with high autistic-like traits find it stressful to interact with sexually attractive strangers due to their poor social and communicative skills, elevation of their stress hormones during these social interactions can impair their motivation or ability to engage in courtship behavior or to initiate or complete sexual interactions. Despite the many potential associations between autistic-like traits, sociosexuality, and sex and stress hormones these relationships have been investigated in only a few studies and with only one measure of baseline hormone concentrations (e.g., Takagishi et al. 2009). No study to date has investigated possible differences in testosterone and/or cortisol reactivity to sexual or socially stressful stimuli in relation to autistic-like traits.

In the present study we investigated autistic-like traits, sociosexuality, and cortisol and testosterone responses to socially stressful and sexually arousing stimuli in male college students. We tested the following hypotheses: 1) that autistic-like traits are associated with restricted sociosexuality, including low number of sexual partners, low short-term mating orientation, and high long-term mating orientation; 2) that individuals with higher autistic-like traits exhibit greater cortisol reactivity to a socially stressful situation than individuals with lower autistic-like traits; 3) that individuals with higher autistic-like traits exhibit lower testosterone reactivity to sexually arousing visual stimuli (especially if they involve a stranger) and higher cortisol reactivity to these stimuli (because they might find them stressful) than individuals with lower 
autistic-like traits; 4) that differential cortisol or testosterone reactivity to socially stressful or sexually arousing stimuli may be one of the physiological mechanisms mediating the association between autistic-like traits and sociosexuality.

\section{Methods}

\section{Participants}

Participants were 107 heterosexual young men recruited on the University of Chicago campus through fliers, mailing lists, or a human subject recruitment website (Sona System). The majority of the participants were students at the University of Chicago. All study participants completed a written informed consent form before participating in the study and were paid \$ 20 after completion of the procedures. Subject recruitment and data collection lasted approximately 12 months; they were stopped when the desired sample size was reached and there was a sharp decline in response rate to the ads due to the end of the academic year. This study and the use of human subjects were approved by the Social Sciences Institutional Review Board of the University of Chicago.

\section{Procedure}

Participants were tested twice, at the same time on two consecutive weekdays, or 1-2 days apart (see Fig. 1 for a diagram of the experimental design). Each day upon arrival, participants were taken to a testing room, where they completed questionnaires for $25 \mathrm{~min}$. At the end of this period they provided a baseline saliva sample and participated in one of two experimental tasks (see below). Another saliva sample was collected immediately after this task, followed by 10 min of additional questionnaires, and a third saliva sample. Upon completion of all procedures, participants were debriefed and given their compensation.

\section{Questionnaires}

An initial demographic survey asked information about participants' age, ethnicity, and marital or relationship status (single or in a relationship). Heterosexual orientation, which was established when participants were first recruited, was also confirmed. Mean

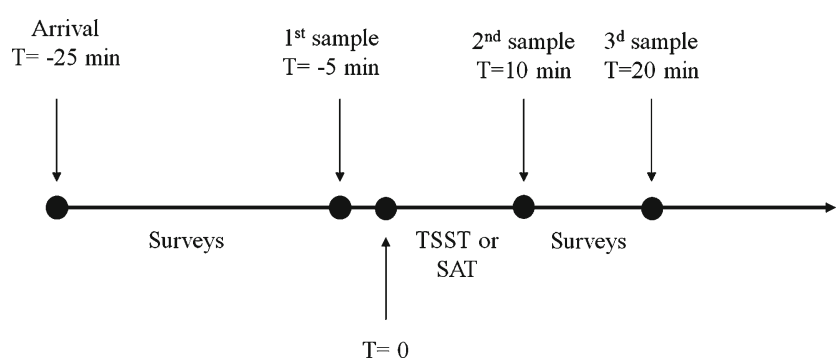

Fig. 1 Timeline for the two experimental sessions 
age was 22.44 years $(S D=3.90)$. Approximately $62 \%$ of study participants were Caucasian, $9 \%$ Hispanic, $9 \%$ African-American, $8 \%$ Asian, and $12 \%$ of other or mixed ethnicities. Among the participants who reported information about their relationship status $(N=97), 48$ were single and 47 in a relationship, 1 was married and 1 was divorced. In terms of previous sexual experience, 16 out of the 107 participants never had sexual intercourse, 21 had intercourse with only one woman, and 70 had intercourse with multiple women. Following the demographic survey, two other questionnaires were administered.

The Autism-Spectrum Quotient (AQ; Baron-Cohen et al. 2001) The AQ is a 50item self-report measure of autistic-like traits in adults with normal IQ. It includes items such as "I prefer to do things with others rather than on my own" or "I tend to have very strong interests that I get upset about if I can't pursue". This questionnaire has been widely used and validated in many studies of children, adolescents, and adults (e.g., Hoekstra et al. 2008; Hurst et al. 2007). In the AQ, four-point Likert-type responses are dichotomized to yield a binary score $(0,1)$ for each item. The total AQ score can range between 0 (no autistic-like traits) to 50 (maximum autistic-like traits). Baron-Cohen et al. (2001) proposed a cut-off score of 32, above which individuals are likely to meet the diagnostic criteria for ASD, while Woodbury-Smith et al. (2005) showed that the AQ has good discriminative validity and good screening properties at a threshold score of 26. In our study, Cronbach's alpha for the total AQ was 0.66. This is typical for the AQ (e.g., Baron-Cohen et al. 2001). Since some studies recommend using the four-point scores instead of the binary score (e.g., Hoekstra et al. 2008), we calculated the total AQ scores with both methods and since these scores were highly correlated $(\mathrm{r}=.90, p<0.001)$, we chose to use the scores obtained with the binary score coding method for data analyses. The AQ comprises five subscales: communication, social skills, attention switching, imagination, and attention to detail. Preliminary data analyses showed that the $5 \mathrm{AQ}$ subscales were only poorly intercorrelated and that none of the correlations between the individual subscales and the sociosexuality or physiological variables was statistically significant. Therefore, only results concerning the total AQ score are reported.

\section{The Multidimensional Sociosexual Inventory (MSOI; Jackson and Kirkpatrick}

2007) The MSOI is a 20 -item measure of sociosexual orientation and behavior. It is comprised of two attitudinal scales with 7-point Likert-type items that measure shortterm mating orientation (STMO; 10 items; $\alpha=.93$ ) and long-term mating orientation (LTMO; 7 items, $\alpha=.88$ ), and one scale measuring past sexual experience (PSE; 3 items, $\alpha=$.74). The STMO and LTMO include items such as "How many partners do you foresee having sexual intercourse with during the next five years?" and "I would have to be closely attached to someone (both emotionally and psychologically) before I could feel comfortable and fully enjoy having sex with him or her". The PSE includes three questions about the lifetime number of sexual partners, the lifetime number of casual sexual partners, and the number of sexual partners in the previous year. In this study, in addition to using the PSE score for data analyses, we also present results concerning one of its components, the lifetime number of sexual partners. Means and standard deviations for the sociosexuality measures were as follows: STMO, mean= $4.12, S D=.85$; LTMO, mean=4.89, $S D=.50$; PSE, mean=3.67, $S D=6.46$; number of 
lifetime sexual partners, mean $=6.44, S D=11.61$. The PSE measure was highly skewed and therefore all data were log-transformed. The LTMO was not significantly correlated with STMO $(\mathrm{r}=-.13, p=.18)$ or PSE $(\mathrm{r}=-.06, p=.55)$. STMO and PSE were positively correlated $(\mathrm{r}=.45, p<.001)$.

\section{Experimental Tasks}

On the first day of testing, subjects underwent the Trier Social Stress Test; on the second day, they underwent a Sexual Arousal Test of approximately equal duration.

Trier Social Stress Test (Kirschbaum et al. 1993) The TSST is a broadly used, standardized task to study hormonal responses to mild psychosocial stress in a laboratory setting. In the current study, the experimenter explained to each participant that he would have 3 min to prepare a 5-min presentation about himself for a mock job interview. Each presentation took place in front of a "selection committee" composed of two unfamiliar confederates ("judges") trained to maintain neutral facial expressions and provide no positive feedback to the participant. Each participant was informed that he must keep speaking for $5 \mathrm{~min}$ and that the presentation would be video-recorded for subsequent analyses of content and non-verbal behavior. If the participant ever stopped speaking before the $5 \mathrm{~min}$ were up, the judges waited in silence for the participant to resume or otherwise prompted him to continue. If the participant again stopped speaking, one of the judges asked one of several standardized questions (e.g., "What do you think about teamwork?"). Upon completing the 5-min speech, the judges asked each participant to perform a difficult arithmetic calculation (i.e., serially subtracting the number 17 from 2023) out loud for another $5 \mathrm{~min}$ or until he reached zero. Anytime the participant made a mistake, he was notified and asked to restart from the beginning. After this task, the confederates thanked the participant and left the room.

Sexual Arousal Test Each participant was set up in front of a computer in his testing room and left alone, with the door closed, to watch a 12-min video clip with explicit erotic content (see Carani et al. 1990). The video was ethically produced by a company called Twistys.com and is freely available on a website managed by the Pornhub Network. The video showed an attractive young woman performing a strip-tease and masturbating. Before and after the sexual arousal test, participants interacted only with male experimenters, to avoid the possible confounding influence of interacting with women.

\section{Saliva Samples and Hormonal Assays}

Five minutes before, immediately after, and 10 min after each experimental task, participants were asked to provide a saliva sample; thus a total of 6 samples were collected for each participant over a 2-day period. All saliva samples were collected between 12:30 PM and 4:30 PM, as previous studies have shown that afternoon hormone levels, although lower than morning levels, are more stable and therefore better suited for studies of social endocrinology (e.g., Gray et al. 2004). Saliva was 
collected by passive drool into plastic tubes. Saliva samples were stored in a refrigerator at $-20{ }^{\circ} \mathrm{F}$ and assayed for cortisol and testosterone using kits provided by the manufacturer (Salimetrics Inc.). For cortisol, the intra-assay CV based on optic density (OD) was $2.18 \%$. The intra-assay CV based on concentration was $7.56 \%$. The inter-assay $\mathrm{CV}$ was $9 \%$. For testosterone, the intra-assay CV based on optic density (OD) was $2.16 \%$. The intra-assay CV based on concentration was $8.23 \%$. The inter-assay CV was $10.53 \%$.

Cortisol and testosterone concentrations were not normally distributed and therefore all hormonal data were log-transformed. Measures of baseline cortisol and testosterone levels were obtained from the first saliva sample (baseline) collected on Day 1 and Day 2 of the experimental procedures. First, we calculated the residuals of the regression predicting baseline hormone levels for each day separately, after controlling for time since wake-up (time elapsed since waking up in the morning) and food or drink consumption within $1 \mathrm{~h}$ prior to the sample. Then, we calculated the mean between the residual scores of Day 1 and Day 2. Post-task peak cortisol and peak testosterone were identified as the highest between the two post-task cortisol and testosterone concentrations, respectively (for the use of a similar procedure, see BuskeKirschbaum et al. 2003; Oswald et al. 2006; Wust et al. 2004) Delta cortisol and delta testosterone were calculated as the difference between the log-transformed post-task peak and baseline levels. The amount and pattern of hormone secretion during the test were assessed by calculating the Area Under the Curve with respect to Increase (AUCI) (e.g., Fekedulegn et al. 2007). The AUC-I is derived as the area under the curve above the baseline value minus the area under the curve below the baseline value. Studies have shown that the sign and magnitude of AUC-I are related to the profile and the rate of change of the cortisol secretion over time (e.g., Fekedulegn et al. 2007).

\section{Statistical Analyses}

Pearson's bivariate correlations were used to investigate possible associations between the variables under study. Hormonal responses to the TSST and the SAT were assessed using linear mixed models with two repeated measures (log transformed baseline and post-task peak hormonal values) modeled with an autoregressive covariance structure. A second model was then used to examine the effects of the AQ on hormones and its possible interaction with sampling time. Time since wake-up was entered in both models as the covariate. Food or drink consumption within one hour from the experimental session was entered as a covariate in the model assessing cortisol. The goodness-of-fit between the two models was based on the Deviance-Based Hypothesis Test (Singer and Willett 2003).

Regression analyses were used to evaluate hormonal measures as possible mediating variables in the association between the AQ and sociosexuality measures. These mediation analyses were performed using the Preacher and Hayes bootstrapping method and Hayes's PROCESS macro for SPSS (Hayes 2013). Bootstrapping involves repeated random sampling (in this case we used 5000 bootstrap samples) with replacement in order to derive $95 \%$ confidence intervals to assess the significance of the indirect effects of interest. A confidence interval that did not overlap with zero was considered an indicator of a statistically significant mediation. Where possible, the kappa squared $\left(\mathrm{K}^{2}\right)$ (Preacher and Kelley 2011) was used as a measure of effect size for 
the indirect effect. $\kappa^{2}$ is a standardized measure bounded between 0 and 1 and independent of sample size, which has similar effect size interpretation as the squared correlation coefficient $\mathrm{R}^{2}$, where a 0.01 would be considered a small, a 0.09 medium and 0.22 a large effect size.

All statistical analyses were carried out with SPSS 22. $P$ values $<.05$ were considered statistically significant.

\section{Results}

\section{AQ and age, Ethnicity, Relationship Status, Sociosexuality, and Baseline Hormone Levels}

Total AQ scores ranged between 6 and 31 and were normally distributed in the subject population (mean=18.01, SD=5.32). Only two individuals had AQ scores above 30 . There was no significant correlation between AQ scores and age $(\mathrm{r}=.01, p=.92)$, or significant differences in AQ scores in relation to ethnicity $\left(\mathrm{F}_{9,97}=.99, p=.45\right)$ or relationship status $(\mathrm{t}=.16, \mathrm{df}=97, p=.87)$ (Table 1$)$.

Study participants scoring higher in the AQ reported significantly lower Short-Term Mating Orientation ( $\mathrm{r}=-.27, p<.01$; Fig. 3a), lower Past Sexual Experience $(\mathrm{r}=-.38$, $p<.001)$ and lower number of lifetime sex partners $(\mathrm{r}=-.39, p<.001)$. There was no significant correlation between $\mathrm{AQ}$ and Long-Term Mating Orientation $(\mathrm{r}=-.16, p=$ 0.09). There was no significant correlation between AQ scores and baseline cortisol levels $(\mathrm{r}=.01, p=.90)$ or between AQ scores and baseline testosterone levels $(\mathrm{r}=.04$; $p=.82)$.

\section{AQ and Hormonal Responses to the Trier Social Stress Test}

Across all study participants, cortisol was significantly higher at the post-task peak compared to baseline (baseline $=0.19 \pm 0.01 \mu \mathrm{g} / \mathrm{dl}$, peak $=0.40 \pm 0.02, \mathrm{~F}_{1,104}=102.71$, $p<.001$ ), indicating that, as expected, cortisol levels increased significantly in response to the experimental procedure (Fig. 2a). AQ significantly influenced overall cortisol secretion during the test: specifically, overall cortisol output was higher in participants with higher AQ than in those with lower AQ $\left(\gamma_{03}=.014 \pm .004, \mathrm{t}=3.35, p=.001\right.$; see model 2, Table 2). There was no significant correlation between AQ scores and delta cortisol $(\mathrm{r}=.14, p=.15)$. However, there was a significant positive correlation between AQ scores and AUC-I $(\mathrm{r}=.20, p<.05)$, indicating that, consistent with our predictions, participants with higher AQ secreted more cortisol in response to the test (Fig. 2c). Therefore, although the difference between post-task peak and baseline cortisol was not significantly correlated with the AQ, the overall amount of cortisol produced in response to the test (after controlling for the baselines) was predicted by the AQ.

Similar to cortisol, testosterone increased significantly during the Trier Social Stress Test $\left(\right.$ baseline $=145.40 \pm 4.32 \mathrm{pg} / \mathrm{ml}$, peak $\left.=161.17 \pm 4.72, \mathrm{~F}_{1,105}=68.69, p<.001\right)$. AQ did not significantly influence overall testosterone secretion during the test $(\mathrm{F}$, $1,105=.03, p=.78)$. The results of the regression analyses were confirmed by the lack of significant correlations between AQ and delta testosterone $(\mathrm{r}=.01, p=.90)$, and between AQ and AUC-I ( $\mathrm{r}=.07, p=.48)$. 
Table 1 Data on age, AQ scores, three measures of sociosexuality (STMO, LTMO, and PSE), and cortisol and testosterone baseline levels for all study participants and for relationship status and ethnicity subgroups of participants

\begin{tabular}{|c|c|c|c|c|c|c|c|}
\hline & Age & AQ & STMO & LTMO & PSE & $\begin{array}{l}\text { Cortisol } \\
\text { baseline }\end{array}$ & $\begin{array}{l}\text { Testosterone } \\
\text { baseline }\end{array}$ \\
\hline All $(N=107)$ & $\begin{array}{l}22.44 \\
(0.37 ; 107)\end{array}$ & $\begin{array}{l}18.01 \\
(0.51 ; 107)\end{array}$ & $\begin{array}{l}4.12 \\
(0.08 ; 104)\end{array}$ & $\begin{array}{l}4.89 \\
(0.05 ; 104)\end{array}$ & $\begin{array}{l}3.67 \\
(0.64 ; 104)\end{array}$ & $\begin{array}{l}0.20 \\
(0.00 ; 106)\end{array}$ & $\begin{array}{l}145.25 \\
(4.21 ; 106)\end{array}$ \\
\hline \multicolumn{8}{|l|}{$\begin{array}{l}\text { Relationship } \\
\text { status }\end{array}$} \\
\hline Single & $\begin{array}{l}22.85 \\
(0.65 ; 48)\end{array}$ & $\begin{array}{l}17.83 \\
(0.72 ; 48)\end{array}$ & $\begin{array}{l}4.24 \\
(0.11 ; 48)\end{array}$ & $\begin{array}{l}4.93 \\
(0.06 ; 48)\end{array}$ & $\begin{array}{l}4.94 \\
(1.25 ; 48)\end{array}$ & $\begin{array}{l}0.22 \\
(0.01 ; 47)\end{array}$ & $\begin{array}{l}148.60 \\
(7.06 ; 48)\end{array}$ \\
\hline Partner & $\begin{array}{l}22.70 \\
(0.50 ; 47)\end{array}$ & $\begin{array}{l}17.65 \\
(0.80 ; 47)\end{array}$ & $\begin{array}{l}4.03 \\
(0.12 ; 47)\end{array}$ & $\begin{array}{l}4.90 \\
(0.08 ; 47)\end{array}$ & $\begin{array}{l}2.68 \\
(0.44 ; 47)\end{array}$ & $\begin{array}{l}0.18 \\
(0.01 ; 47)\end{array}$ & $\begin{array}{l}140.59 \\
(5.72 ; 47)\end{array}$ \\
\hline \multicolumn{8}{|l|}{ Ethnicity } \\
\hline Caucasian & $\begin{array}{l}22.86 \\
(0.47 ; 66)\end{array}$ & $\begin{array}{l}18.37 \\
(0.65 ; 66)\end{array}$ & $\begin{array}{l}5.05 \\
(0.11 ; 64)\end{array}$ & $\begin{array}{l}4.90 \\
(0.06 ; 64)\end{array}$ & $\begin{array}{l}4.02 \\
(0.97 ; 64)\end{array}$ & $\begin{array}{l}0.20 \\
(0.01 ; 65)\end{array}$ & $\begin{array}{l}143.80 \\
(5.56 ; 65)\end{array}$ \\
\hline $\begin{array}{l}\text { African- } \\
\text { American }\end{array}$ & $\begin{array}{l}24.10 \\
(1.65 ; 10)\end{array}$ & $\begin{array}{l}16.90 \\
(1.05 ; 10)\end{array}$ & $\begin{array}{l}4.30 \\
(0.25 ; 9)\end{array}$ & $\begin{array}{l}5.12 \\
(0.10 ; 9)\end{array}$ & $\begin{array}{l}2.51 \\
(0.61 ; 9)\end{array}$ & $\begin{array}{l}0.15 \\
(0.01 ; 9)\end{array}$ & $\begin{array}{l}133.18 \\
(16.49 ; 9)\end{array}$ \\
\hline Hispanic & $\begin{array}{l}20.60 \\
(0.45 ; 10)\end{array}$ & $\begin{array}{l}17.30 \\
(1.84 ; 10)\end{array}$ & $\begin{array}{l}4.17 \\
(0.27 ; 10)\end{array}$ & $\begin{array}{l}4.84 \\
(0.14 ; 10)\end{array}$ & $\begin{array}{l}5.46 \\
(1.89 ; 10)\end{array}$ & $\begin{array}{l}0.23 \\
(0.01 ; 10)\end{array}$ & $\begin{array}{l}157.29 \\
(16.44 ; 10)\end{array}$ \\
\hline Asian & $\begin{array}{l}21.11 \\
(1.22 ; 9)\end{array}$ & $\begin{array}{l}20 \\
(2.21 ; 9)\end{array}$ & $\begin{array}{l}4.22 \\
(0.27 ; 9)\end{array}$ & $\begin{array}{l}4.76 \\
(0.21 ; 9)\end{array}$ & $\begin{array}{l}1.00 \\
(0.22 ; 9)\end{array}$ & $\begin{array}{l}0.21 \\
(0.03 ; 9)\end{array}$ & $\begin{array}{l}152.78 \\
(7.25 ; 9)\end{array}$ \\
\hline Others & $\begin{array}{l}21.33 \\
(1.20 ; 12)\end{array}$ & $\begin{array}{l}16.08 \\
(1.43 ; 12)\end{array}$ & $\begin{array}{l}4.21 \\
(0.22 ; 12)\end{array}$ & $\begin{array}{l}4.82 \\
(0.15 ; 12)\end{array}$ & $\begin{array}{l}3.19 \\
(0.83 ; 12)\end{array}$ & $\begin{array}{l}0.19 \\
(0.02 ; 12)\end{array}$ & $\begin{array}{l}147.56 \\
(9.77 ; 12)\end{array}$ \\
\hline
\end{tabular}

Data are presented as mean values $(S E$ and $\mathrm{N})$. Cortisol $(\mu \mathrm{g} / \mathrm{dl})$ and testosterone $(\mathrm{pg} / \mathrm{ml})$ are given as the average of the raw values of the first samples collected during the two experimental days

\section{AQ and Hormonal Responses to the Sexual Arousal Test}

There was no significant difference between the post-task cortisol and the baseline cortisol (baseline $=0.21 \pm 0.01$, peak $=0.22 \pm 0.01 ; \mathrm{F}_{1,102}=.27, p=.60$ ), indicating that the sexual arousal test was not associated with a significant change in cortisol. The main effect of AQ was nonsignificant $\left(\mathrm{F}_{1,102}=1.02, p=.31\right)$, indicating that the AQ did not affect overall cortisol production or change in cortisol over time. However, AQ was significantly correlated with delta cortisol $(\mathrm{r}=.20, p<.05)$ and with the AUC-I $(\mathrm{r}=.25$, $p=.01$ ), indicating that participants with higher AQ showed a greater increase in cortisol relative to baseline, and generally produced more cortisol during the test.

Across all study participants, testosterone was higher at post-task peak compared to baseline (baseline $=143.90 \pm 4.71$, peak $=155.20 \pm 5.08, \mathrm{~F}_{1,103}=66.98, p<.001$; Fig. $2 \mathrm{~b}$ ). Thus, consistent with our hypothesis, exposure to sexual stimuli was associated with an increase in testosterone. The main effect of $\mathrm{AQ}$ was nonsignificant $\left(\mathrm{F}_{1,103}=.02, p=.88\right)$. There was a significant interaction between AQ and temporal changes in testosterone $\left(\mathrm{F}_{1,103}=5.58, p<.05\right)$, suggesting that changes in testosterone between baseline and post-task are moderated by AQ. The results of the regression analyses were confirmed by a significant positive correlation between AQ and AUC-I ( $\mathrm{r}=0.21, p<.05$, Fig. $2 \mathrm{~d})$. The correlation between AQ and delta testosterone was positive too, and approached statistical significance $(\mathrm{r}=.18, p=.06)$. Since we had predicted that participants with 

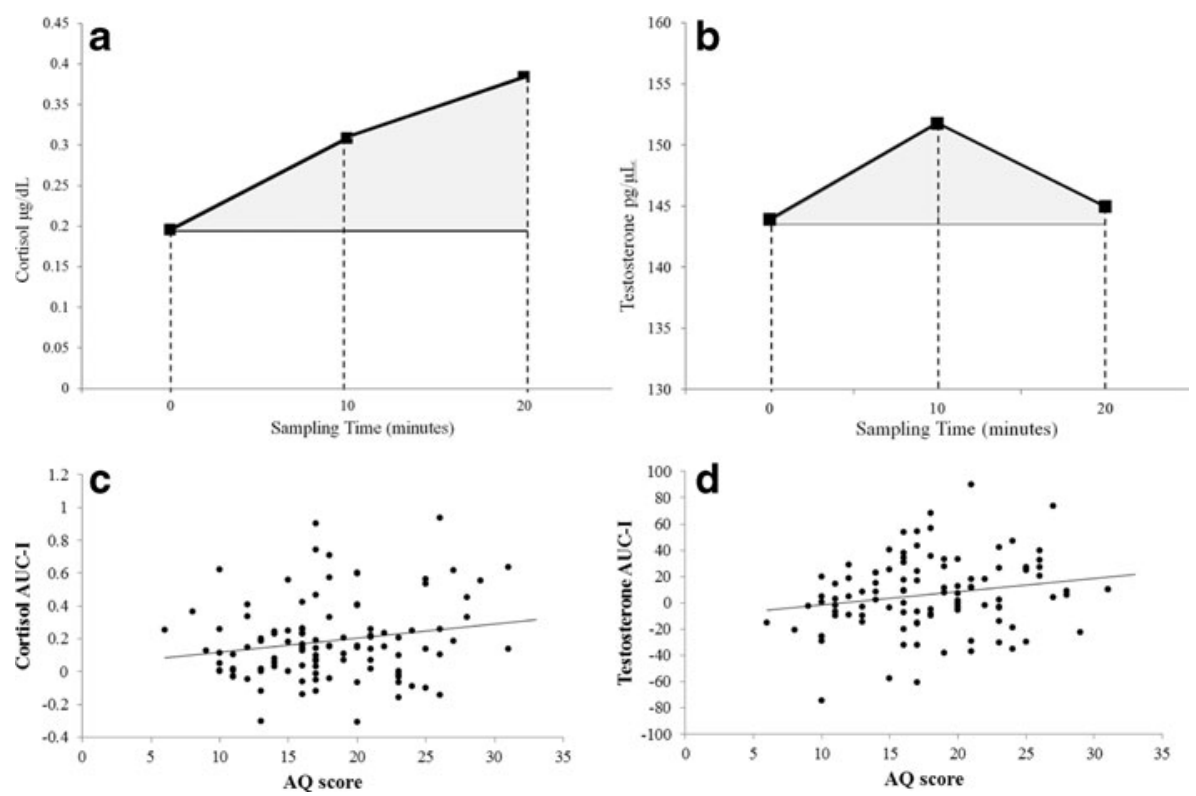

Fig. 2 a, b Shaded area represents the area under the curve with respect to increase (AUC-I) for cortisol during the TSST (a) and for testosterone during the SAT (b). c Correlation between the AQ scores and cortisol AUC-I during the TSST. d Correlation between the AQ scores and testosterone AUC-I during the SAT

higher AQ scores would show a lower increase in testosterone in response to sexual stimuli, the above results were opposite to those we predicted.

\section{Correlations Between Hormonal Variables}

There were significant positive correlations between baseline cortisol and testosterone concentrations measured on Day 1 and those measured on Day 2 (cortisol: $r=.54$, $p<.001$; testosterone: $\mathrm{r}=.75 ; p<.0001)$. Across all individuals, there was a significant positive correlation between baseline cortisol and baseline testosterone levels $(\mathrm{r}=.40$, $p<.001)$. Thus, participants who had higher baseline cortisol also had higher baseline testosterone. In both the Trier Social Stress Test and the Sexual Arousal Test, participants who had a higher increase in cortisol (delta cortisol) also had a higher increase in testosterone (delta testosterone) (TSST, $\mathrm{r}=.35, p<.001$; SAT, $\mathrm{r}=.39, p<.001$ ).

There was a positive but weak and nonsignificant correlation between delta cortisol in the TSST and delta cortisol in the SAT $(\mathrm{r}=.10 ; p=.31)$. There was, however, a stronger and significant positive correlation between the cortisol AUC-I in the TSST and the cortisol AUC-I in the SAT $(\mathrm{r}=.27, p<.01)$, indicating that the individuals who secreted more cortisol during the TSST also secreted more cortisol during the SAT. With the exception of baseline levels, testosterone measures in the TSST were not significantly correlated with similar testosterone measures in the SAT $(\mathrm{r}=.10, p=.31$; $\mathrm{r}=.09, p=.37$, for delta testosterone and AUC-I, respectively).

Taken together, these results suggest that 1 ) there are marked individual quantitative differences in hormone secretion, which are consistent over time (Day 1 and Day 2), across hormones (cortisol and testosterone), and across experimental conditions (TSST 
Table 2 Model comparisons for the linear mixed models

\begin{tabular}{|c|c|c|c|c|c|c|c|c|}
\hline & \multicolumn{4}{|l|}{ Cortisol } & \multicolumn{4}{|c|}{ Testosterone } \\
\hline & \multicolumn{2}{|l|}{ TSST } & \multicolumn{2}{|l|}{ SAT } & \multicolumn{2}{|l|}{ TSST } & \multicolumn{2}{|l|}{ SAT } \\
\hline & Model 1 & Model 2 & Model 1 & Model 2 & Model 1 & Model 2 & Model 1 & Model 2 \\
\hline Intercept & $\begin{array}{l}-0.33^{* *} \\
(0.06)\end{array}$ & $\begin{array}{l}-0.55^{* *} \\
(0.09)\end{array}$ & $\begin{array}{l}-0.52^{* *} \\
(0.07)\end{array}$ & $\begin{array}{l}-0.62^{* *} \\
(0.09)\end{array}$ & $\begin{array}{l}2.27^{* *} \\
(0.39)\end{array}$ & $\begin{array}{l}2.27^{* *} \\
(0.05)\end{array}$ & $\begin{array}{l}2.28^{* * *} \\
(0.04)\end{array}$ & $\begin{array}{l}2.26^{* *} \\
(0.06)\end{array}$ \\
\hline Time $(2=0)$ & $\begin{array}{l}-0.30^{* *} \\
(0.03)\end{array}$ & $\begin{array}{l}-0.14 \\
(0.10)\end{array}$ & $\begin{array}{l}-0.01 \\
(0.01)\end{array}$ & $\begin{array}{l}0.06 \\
(0.04)\end{array}$ & $\begin{array}{l}-0.04^{* *} \\
(0.01)\end{array}$ & $\begin{array}{l}-0.03^{\dagger} \\
(0.02)\end{array}$ & $\begin{array}{l}-0.03^{* *} \\
(0.00)\end{array}$ & $\begin{array}{l}-0.00 \\
(0.01)\end{array}$ \\
\hline Time wake up & $\begin{array}{l}-0.03^{* *} \\
(0.01)\end{array}$ & $\begin{array}{l}-0.03^{* *} \\
(0.01)\end{array}$ & $\begin{array}{l}-0.04^{* *} \\
(0.01)\end{array}$ & $\begin{array}{l}-0.04^{* *} \\
(0.01)\end{array}$ & $\begin{array}{l}-0.02^{* *} \\
(0.01)\end{array}$ & $\begin{array}{l}-0.02^{* *} \\
(0.01)\end{array}$ & $\begin{array}{l}-0.02^{* *} \\
(0.01)\end{array}$ & $\begin{array}{l}-0.02^{* *} \\
(0.01)\end{array}$ \\
\hline Food/drink & $\begin{array}{l}0.02 \\
(0.04)\end{array}$ & $\begin{array}{l}0.02 \\
(0.04)\end{array}$ & $\begin{array}{l}0.09^{\dagger} \\
(0.06)\end{array}$ & $\begin{array}{l}0.10^{\dagger} \\
(0.06)\end{array}$ & $\begin{array}{l}0.04 \\
(0.03)\end{array}$ & $\begin{array}{l}0.04 \\
(0.03)\end{array}$ & $\begin{array}{l}0.01 \\
(0.03)\end{array}$ & $\begin{array}{l}0.01 \\
(0.03)\end{array}$ \\
\hline $\mathrm{AQ}$ & & $\begin{array}{l}0.01^{* *} \\
(0.01)\end{array}$ & & $\begin{array}{l}0.01 \\
(0.00)\end{array}$ & & $\begin{array}{l}0.00 \\
(0.00)\end{array}$ & & $\begin{array}{l}0.00 \\
(0.00)\end{array}$ \\
\hline AQ x Time & & $\begin{array}{l}-0.01 \\
(0.01)\end{array}$ & & $\begin{array}{l}-0.00 \\
(0.00)\end{array}$ & & $\begin{array}{l}-0.00 \\
(0.00)\end{array}$ & & $\begin{array}{l}-0.00^{*} \\
(0.00)\end{array}$ \\
\hline Deviance & -4.30 & $-15.64^{*}$ & -152.13 & -156.02 & -470.94 & -471.21 & -491.92 & $-497.37^{*}$ \\
\hline
\end{tabular}

Note. The test for goodness-of-fit was based on the Deviance-Based Hypothesis test, which is based on a $\chi^{2}$ distribution with degrees of freedom equal to the number of parameters difference between the two nested models. Coefficients are based on log-transformed values of cortisol and testosterone (the AUC-I measures). Model 1=effect of test on hormone concentrations (post-test vs baseline levels). Model 2=Effect of test and AQ on hormone concentrations. Time $=$ times at which samples were collected. Time wake up $=$ time since wake-up. Standard deviation (SE) in italic. ${ }^{\dagger} p<0.10 ;{ }^{*} p<0.05 ;{ }^{* *} p<0.01$

and SAT); 2) individual differences in cortisol secretion across the TSST and the SAT were generally correlated, whereas individual differences in testosterone secretion across these two tests were not.

\section{Relationships Between Hormonal Variables and Sociosexuality Measures and Mediation Analysis With the AQ}

Delta cortisol and the cortisol AUC-I in the TSST were significantly negatively correlated with Short-Term Mating Orientation (delta cortisol, $\mathrm{r}=-.20, p<.01$; AUC-I, $r=-.22, p<.05$; Fig. $3 b$ ), but not with the other sociosexuality measures. Thus, the study participants who were less oriented toward short-term mating showed higher cortisol responses to the TSST. Neither delta testosterone $(\mathrm{r}=-.13, p=.19)$ nor testosterone AUC-I in the TSST $(\mathrm{r}=-.07, p=.44)$ was significantly correlated with any sociosexuality measures. There were no significant correlations between hormonal responses in the SAT and any sociosexuality measures.

A mediation analysis was performed to test whether cortisol reactivity to the TSST was the variable mediating the significant negative association between AQ scores and Short-Term Mating Orientation. Although the analysis using the delta cortisol did not produce statistically significant results, the analysis with the AUC-I did: the overall model testing the effect of AQ and AUC-I on Short-Term Mating Orientation was 

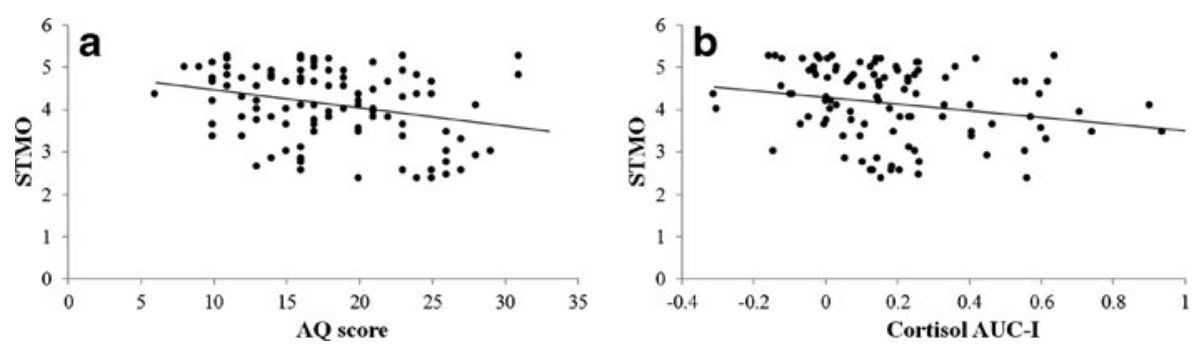

Fig. 3 a Correlation between AQ scores and STMO (short-term mating orientation) scores; b Correlation between cortisol AUC-I during the TSST and STMO scores

significant $\left(\mathrm{R}^{2}=.04, p<.05\right)$ and the indirect effect was significant as well (Fig. 4; $\mathrm{ab}=$ $\left.-.006,95 \% \mathrm{CI}[-.01,-.0003], \mathrm{K}^{2}=.03\right)$, suggesting that the higher cortisol secretion in response to psychosocial stress mediates the association between high AQ and low Short-Term Mating Orientation.

\section{Discussion}

Heterosexual male college students with more autistic-like traits reported lower shortterm mating orientation and less previous sexual experience, including lower number of lifetime sexual partners, when compared to individuals with fewer autistic-like traits. While it is not clear whether less previous sexual experience in individuals with higher AQ reflects their choice (i.e., they are not as interested in sexual activity as individuals with lower AQ) or a constraint (i.e., they have fewer opportunities to engage in sexual activity), lower short-term mating orientation reflects a lower preference for casual sexual activity. These results replicate those of two previous studies (Del Giudice et al. 2010, 2014) and are generally consistent with the hypothesis that autistic-like traits reflect a slow life history phenotype (Del Giudice et al. 2010). It is also possible, however, that men with high AQ scores do not have a well defined slow life history strategy that entails multiple, coordinated components and that the observed association between high AQ and low STMO represents an isolated effect.

The association between autistic-like traits and long-term mating orientation was not significant. This association, however, has been reported by another study (Del Giudice et al. 2010). The reason for this discrepancy between the results of these studies is unclear. If the association between autistic-like traits and long-term mating orientation also fails to replicate in future studies, one would have to conclude that people with higher autistic-like traits are averse to short-term mating strategies but they do not necessarily self-identify as being more oriented toward longer-term, more monogamous relationships. The STMO and LTMO were not significantly correlated and these scales represent orthogonal dimensions in mating strategies. It is possible that individuals with higher autistic-like traits show an aversion toward short-term mating strategies due to their previous behavioral experiences and their outcomes (e.g., having been frequently rejected by potential short-term mating partners) but this does not necessarily shift their preferences the other way, toward a higher long-term mating orientation, as a result. Their aversion to short-term mating, however, would still make individuals with higher autistic-like traits appealing as potential long-term relationship partners. It is important 


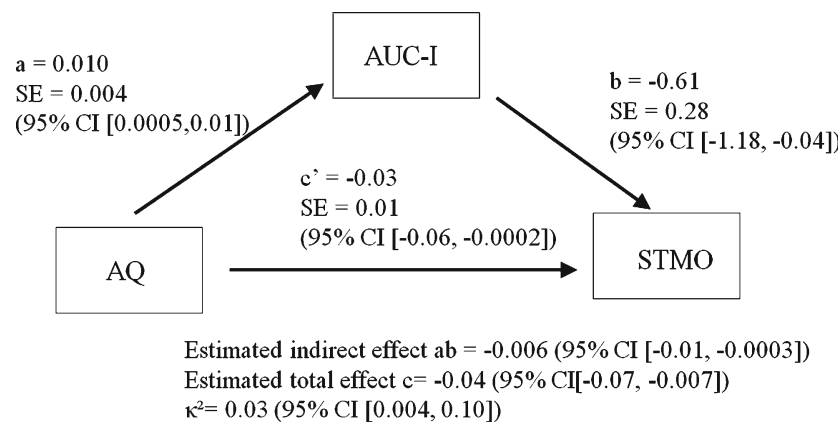

Fig. 4 Mediating role of cortisol AUC-I during the TSST. Path coefficients are unstandardized

to note, however, that no study has yet provided evidence that women have a mating preference for the suite of autistic-like traits per se. It is also possible that if men with higher autistic-like traits achieve high status in their professions, their status is an important component of their appeal as long-term mating partners to women.

We found no significant correlations between $\mathrm{AQ}$ scores and baseline testosterone or cortisol levels. Our study, however, provides the first evidence of AQ-related significant differences in hormonal reactivity to socially stressful and sexually arousing stimuli. The Trier Social Stress Test was effective in causing a significant increase in salivary cortisol concentrations, indicating that study participants perceived the experimental situation as stressful. As previously reported in other experimental stressful situations (e.g., Chichinadze and Chichinadze 2008; Lennartsson et al. 2012), there was also a significant increase in salivary testosterone concentrations. Cortisol and testosterone were positively correlated, and so too were the hormonal responses to both the Trier and the SAT. The correlations between hormones and between hormonal responses to the different tests might reflect physiological interactions between the HPA and the HPG axis or associations between hormonal variables and stable individual characteristics such as personality traits.

There was a main effect of AQ on cortisol secretion, indicating that overall cortisol secretion during the TSST was higher in participants with higher AQ than in those with lower AQ scores. This result was confirmed by a significant positive correlation between AQ scores and the cortisol Area Under the Curve Relative to Increase (AUC-I), which provides a measure of the magnitude and pattern of cortisol change after the baseline. However, it cannot be unequivocally concluded that individuals with higher AQ scores exhibited higher cortisol reactivity to stress because there was no significant interaction between AQ and cortisol change over time or a significant correlation between AQ scores and delta cortisol.

Viewing a video with explicit erotic content was associated with a significant increase in salivary testosterone, but not in cortisol, concentrations. Individuals with higher AQ scores showed higher testosterone reactivity to the test, suggesting that they experienced greater sexual arousal than individuals with lower AQ scores. There was also a significant positive correlation between AQ scores and delta cortisol, suggesting that individuals with high AQ scores experienced an increase in cortisol while those with low AQ scores did not (or a experienced a lower increase). This may suggest that although some individuals were sexually aroused by the video, they also found it, to some extent, stressful. This interpretation is supported by a significant correlation 
between cortisol secretion in the TSST and cortisol secretion in the SAT, suggesting a similarity in individuals' physiological stress responses in the two experimental situations, while no such significant correlation was found for testosterone. This interpretation, however, remains speculative and there are other possible explanations for the correlation between AQ scores and delta cortisol during the SAT.

Cortisol reactivity to the TSST, as assessed through the cortisol AUC-I and the delta cortisol measures, was significantly negatively correlated with short-term mating orientation (STMO). Thus, the study participants who were less oriented toward short-term mating showed higher cortisol responses to the TSST. Since cortisol AUC-I was also correlated with AQ, and AQ and STMO were significantly negatively correlated, we performed a mediation analysis to examine the relationships between these three variables. In this analysis, cortisol AUC-I emerged as a significant mediator of the association between AQ and STMO, suggesting that higher physiological reactivity to psychosocial stress in individuals with higher AQ may be one of the reasons why these individuals are less short-term mating oriented. Differential cortisol reactivity to psychosocial stress can therefore be a physiological mechanism responsible for the different sociosexuality profiles of men on fast and slow life history trajectories (e.g., sexual promiscuity vs monogamy). Since the tendency toward sexual promiscuity that is characteristic of fast life histories requires being comfortable with strangers as potential sexual partners, it makes sense that men with fast life histories do not find it stressful to perform (e.g., as in courtship performances) in front of strangers. In contrast, men with slow life histories may be more reactive to social evaluation threats, such as those elicited in the TSST, and this likely interferes with their ability to court and attract strangers as potential sexual partners.

Individuals with higher AQ scores exhibited higher testosterone responses to visual sexual stimuli than individuals with lower AQ scores. This result was contrary to our expectations and inconsistent with previous suggestions that autistic-like traits may be associated with deficits in sexual arousability (e.g., White et al. 2011). The reason for this difference remains unclear but it may be related to differential exposure to pornography in men with high and low AQ scores. Since testosterone reactivity to visual sexual stimuli was not correlated with any sociosexuality measures, our study provides no hint that physiological sexual arousability may be an important mechanism underlying the association between autistic-like traits and sociosexuality. Instead, our study suggests that stress hormones play an important role in mediating life historyrelated differences in male sociosexuality.

Overall, the results of our study make original and potentially important contributions to different areas of psychological research, including research on the physiological correlates of autistic-like traits in normal populations, research on psychosocial stress and sexual arousal, and research on male life history strategies. Some of the limitations of our approach include the use of an all-male subject population, the use of self-report measures of autistic-like traits and sociosexuality rather than behavioral measures, a relatively small sample size, and the relatively small effects reported in some of our data analyses. Further studies are needed to establish whether out findings can be extrapolated to women, to nonstudent subject populations, and to other experimental or naturalistic settings. We hope that the integrative and interdisciplinary approach taken in this study will stimulate further attempts to test evolutionary hypotheses concerning the functional significance of individual differences in 
personality traits and sociosexuality, while at the same addressing their underlying physiological mechanisms.

\section{References}

Baron-Cohen, S. (2003). The essential difference. New York: Penguin.

Baron-Cohen, S., Wheelwright, S., Skinner, R., Martin, J., \& Clubley, E. (2001). The autism-spectrum quotient (AQ): evidence from asperger syndrome/high-functioning autism, males and females, scientists and mathematicians. Journal of Autism and Developmental Disorders, 31, 5-17.

Buske-Kirschbaum, A., von Auer, K., Krieger, S., Weis, S., Rauh, W., \& Hellhammer, D. (2003). Blunted cortisol responses to psychosocial stress in asthmatic children: a general feature of atopic disease? Psychosomatic Medicine, 65, 806-810.

Carani, C., Bancroft, J., Del Rio, G., Granata, A. R. M., Facchinetti, F., \& Marrama, P. (1990). The endocrine effects of visual erotic stimuli in normal men. Psychoneuroendocrinology, 15, 207-216.

Chichinadze, K., \& Chichinadze, N. (2008). Stress-induced increase of testosterone: contributions of social status and sympathetic activity. Physiology \& Behavior, 94, 595-603.

Del Giudice, M. (2014). An evolutionary life history framework for psychopathology. Psychological Inquiry, $25,261-300$.

Del Giudice, M., Angeleri, R., Brizio, A., \& Elena, M. R. (2010). The evolution of autistic-like and schizotypal traits: a sexual selection hypothesis. Frontiers in Psychology, 1, 41.

Del Giudice, M., Klimczuk, A. C. E., Traficonte, D. M., \& Maestripieri, D. (2014). Autistic-like and schizotypal traits in a life history perspective: diametrical associations with impulsivity, sensation seeking, and sociosexual behavior. Evolution and Human Behavior, 35, 415-424.

Doerr, P., \& Pirke, K. M. (1976). Cortisol-induced suppression of testosterone in normal adult males. Journal of Clinical Endocrinology and Metabolism, 43, 622-629.

Fekedulegn, D. B., Andrew, M. E., Burchfiel, C. M., Violanti, J. M., Hartley, T. A., Charles, L. E., \& Miller, D. B. (2007). Area under the curve and other summary indicators of repeated waking cortisol measurements. Psychosomatic Medicine, 69, 651-659.

Goldey, K. L., \& van Anders, S. (2015). Sexual modulation of testosterone: insights for humans from across species. Adaptive Human Behavior and Physiology, 1(2), 93-123.

Gray, P. B., Campbell, B. C., Marlowe, F. W., Lipson, S. F., \& Ellison, P. T. (2004). Social variables predict between-subject but not day-to-day variation in the testosterone of US men. Psychoneuroendocrinology, 29, 1153-1162.

Happé, F., \& Ronald, A. (2008). The "fractionable autism triad": a review of evidence from behavioural, genetic, cognitive and neural research. Neuropsychology Review, 18, 287-304.

Hayes, A. F. (2013). Introduction to mediation, moderation, and conditional process analysis. A regressionbased approach. New York: Guilford Press.

Hoekstra, R. A., Bartels, M., Verweij, C. J., \& Boomsma, D. I. (2007). Heritability of autistic traits in the general population. Archives of Pediatrics \& Adolescent Medicine, 161, 372-377.

Hoekstra, R. A., Bartels, M., Cath, D. C., \& Boomsma, D. I. (2008). Factor structure, reliability and criterion validity of the Autism-Spectrum Quotient (AQ): a study in Dutch population and patient groups. Journal of Autism and Developmental Disorders, 38, 1555-1566.

Hurst, R. M., Mitchell, J. T., Kimbrel, N. A., Kwapil, T. K., \& Nelson-Gray, R. O. (2007). Examination of the reliability and factor structure of the Autism Spectrum Quotient (AQ) in a non-clinical sample. Personality and Individual Differences, 43, 1938-1949.

Jackson, J. J., \& Kirkpatrick, L. A. (2007). The structure and measurement of human mating strategies: toward a multidimensional model of sociosexuality. Evolution and Human Behavior, 28, 382-391.

Jobe, L. E., \& White, S. W. (2007). Loneliness, social relationships, and a broader autism phenotype in college students. Personality and Individual Differences, 42, 1479-1489.

Kaplan, H. S., \& Gangestad, S. W. (2005). Life history theory and evolutionary psychology. In D. M. Buss (Ed.), Handbook of evolutionary psychology (pp. 68-95). Hoboken: Wiley.

Kirschbaum, C., Pirke, K. M., \& Hellhammer, D. H. (1993). The 'Trier social stress test' - a tool for investigating psychobiological stress responses in a laboratory setting. Neuropsychobiology, 28, 76-81.

Lennartsson, A. K., Kushnir, M. M., Bergquist, J., Billig, H., \& Jonsdottir, I. H. (2012). Sex steroid levels temporarily increase in response to acute psychosocial stress in healthy men and women. International Journal of Psychophysiology, 84, 246-253. 
Maestripieri, D., Klimczuk, A., Traficonte, D., \& Wilson, M. C. (2014). Ethnicity-related variation in sexual promiscuity, relationship status and testosterone levels in men. Evolutionary Behavioral Sciences, 8, 96108.

Oswald, L. M., Zandi, P., Nestadt, G., Potash, J. B., Kalaydjian, A. E., \& Wand, G. S. (2006). Relationships between cortisol responses to stress and personality. Neuropsychopharmacology, 31, 1583-1591.

Preacher, K. J., \& Kelley, K. (2011). Effect size measures for mediation models: quantitative strategies for communicating indirect effects. Psychological Methods, 16, 93-115.

Reser, J. E. (2011). Conceptualizing the autism spectrum in terms of natural selection and behavioral ecology: the solitary forager hypothesis. Evolutionary Psychology, 9, 207-238.

Roney, J. R., \& Simmons, Z. L. (2013). Hormonal predictors of sexual motivation in natural menstrual cycles. Hormones and Behavior, 63, 636-645.

Roney, J. R., Mahler, S. V., \& Maestripieri, D. (2003). Behavioral and hormonal responses of men to brief interactions with women. Evolution and Human Behavior, 24, 365-375.

Shaner, A., Miller, G., \& Mintz, J. (2008). Autism as the low-fitness extreme of a parentally selected fitness indicator. Human Nature, 19, 389-413.

Singer, J., \& Willett, J. B. (2003). Applied longitudinal analysis: modeling change and event occurrence. New York: Oxford University Press.

Stearns, S. C. (1992). The evolution of life histories. Oxford: Oxford University Press.

Takagishi, H., Takahashi, T., Yamagishi, T., Shinada, M., Inukai, K., Tanida, S., Mifune, N., Horita, Y., Hashimoto, H., Yang, Y., \& Kameda, T. (2009). Salivary testosterone levels and autism-spectrum quotient in adults. Neuroendocrinology Letters, 31, 837-841.

Wheelwright, S., Auyeung, B., Allison, C., \& Baron-Cohen, S. (2010). Defining the broader, medium, and narrow autism phenotype among parents using the Autism Spectrum Quotient (AQ). Molecular Autism, 1,10 .

White, S. W., Ollendick, T. H., \& Bray, B. C. (2011). College students on the autism spectrum. Prevalence and associated problems. Autism, 15, 683-701.

Woodbury-Smith, M., Robinson, J., Wheelwright, S., \& Baron-Cohen, S. (2005). Screening adults for asperger syndrome using the AQ: a preliminary study of its diagnostic validity in clinical practice. Journal of Autism and Developmental Disorders, 35, 331-335.

Wust, S., van Rossum, E. F. C., Federnko, I. S., Koper, J. W., Kumsta, R., \& Hellhammer, D. H. (2004). Common polymorphisms in the glucocorticoid receptor gene are associated with adrenocortical responses to psychosocial stress. The Journal of Clinical Endocrinology and Metabolism, 89, 565-573. 